\title{
MOTION ARTIFACTS IN QUANTITATIVE MAGNETIC RESONANCE IMAGING
}

\author{
Alex M. Aisen, M.D., Gary M. Glazer, M.D., Paul L. Carson, Ph.D., \\ and David O. Hearshen, Ph.D. \\ Department of Radiology, The University of Michigan, Ann Arbor, Michigan 48109
}

\begin{abstract}
Several investigators have emphasized the potential value of quantitative relaxation times in the assessment of diseases. In performing such measurements using the spin-echo technique, we have encountered several anomalous results, whereby the intensity of the organ parenchyma on second-echo images is greater than on first echo images. This is most likely a result of respiratory motion, and it occurs only rarely. Several volunteers were studied before and after exercise to see if respiratory motion could reproduce the anomalous intensity reverse; a reversal of intensities in renal parenchyma was observed in two of five individuals. We conclude that respiratory motion artifacts will seriously limit quantitative magnetic resonance imaging of the upper abdomen if respiratory gating during imaging is not used.
\end{abstract}

Keywords: Magnetic resonance imaging, Relaxation times, Motion artifact.

It is well known that magnetic resonance imaging (MRI) is sensitive to motion due to the movement of protons into or out of the imaging volume between the RF pulses of the imaging sequence. Motion can influence the appearance of NMR images; when twodimensional Fourier reconstruction of the data is used, motion artifact typically takes the form of aliasing; multiple "ghost" images appear in the direction of the phase-encoding gradient. ${ }^{1,2}$ These are generally easy to recognize; however, more subtle effects can occur and prove difficult to visually identify. In this preliminary report, we describe discrepancies in MRI intensity measurements of the kidney, which are likely the result of organ motion. As a result of this phenomena, inaccuracies may be produced in relaxation time measurements.

\section{MATERIALS AND METHODS}

The University of Michigan Magnetic Resonance Imager is a Diasonics MT/S system, based on a superconducting magnet operating at 3.5 kilogauss. Detailed descriptions of the unit have appeared elsewhere, ${ }^{3}$ but several pertinent aspects of its operation will be reviewed. The primary pulse sequence used is a dual spin-echo sequence, whereby two images at each anatomic level are acquired simultaneously, one with
Table 1. Magnetic resonance intensities for 3 patients

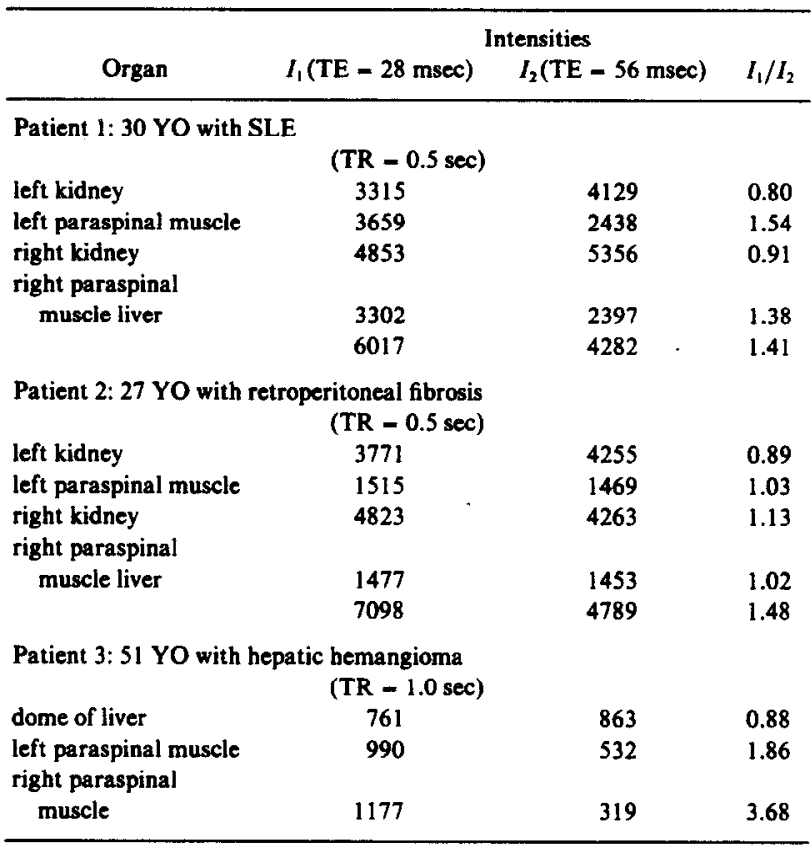

an echo-delay time (TE) of $28 \mathrm{msec}$, the second with a TE of $56 \mathrm{msec}$. Images are obtained using a multiplanar technique, with adjacent parallel planes excited sequentially during the imaging process. The number
RECEIVED 3/8/85; ACCEPTED $11 / 11 / 85$.

Address reprint requests to Alex M. Aisen, M.D., Univer- sity Hospitals, Department of Radiology, Box 0030, Ann Arbor, MI 48109. 


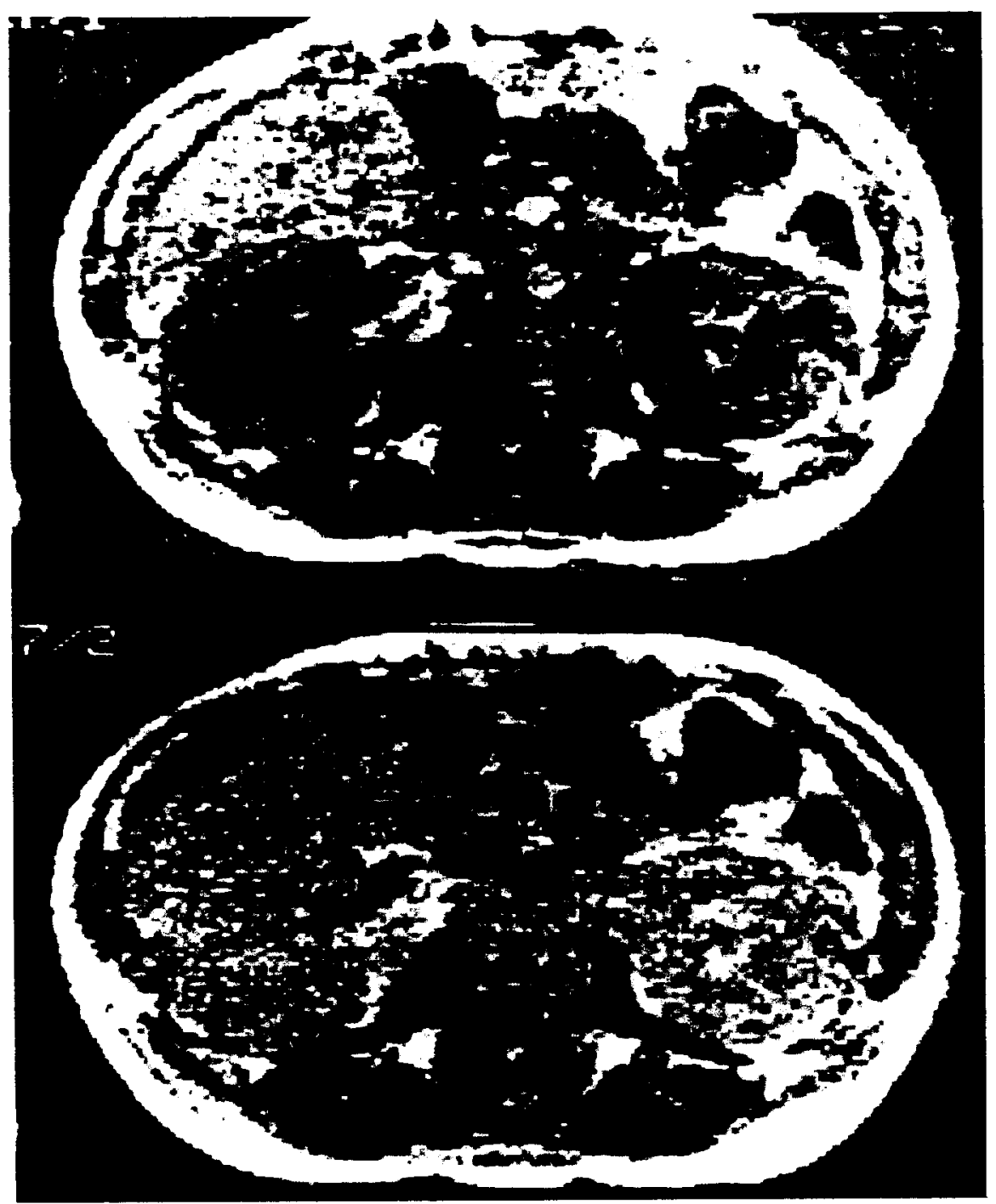

Fig. 1. First echo (top) and second echo (bottom) images of patient with SLE. Intensity normalization is suppressed, so that intensities on the two images can be compared directly. $T R=0.5 \mathrm{sec}$., and $T E=28$ and $56 \mathrm{msec}$, respectively. A blood flow artifact is incidentally noted in the aorta.

of images produced during a single imaging sequence is dictated by the repetition time (TR); for example, 5 sequential planes are imaged using a TR of $0.5 \mathrm{sec}$ in about $4 \frac{1}{2}$ minutes time, while 20 are produced in 18 minutes when a TR of $2.0 \mathrm{sec}$ is employed.

The intensity of the signal in each pixel of the MR image is given approximately by the formula

$$
I=H F(v) \exp (-\mathrm{TE} / T 2) \exp (1-\mathrm{TR} / T 1)
$$

where $H$ is the local tissue hydrogen density, $F(v)$ is a flow function which is a constant in the absence of motion, TE and TR are known timing intervals used by the imager, and $T 1$ and $T 2$ are the local tissue relaxation times. ${ }^{3}$ Since two images in each plane are always acquired simultaneously, it is possible to compute $T 2$ using the equation $T 2=28 \mathrm{msec} / \ln \left(I_{1} / I_{2}\right)$, derived from the above intensity relation, where $I_{1}$ and $I_{2}$ are the local image intensities at 28 and $56 \mathrm{msec}$, respectively. Since $T 2$ must be a positive number, the ratio of $I_{1}$ to $I_{2}$ must always be greater than one, that is the intensity at each point in the "second-echo" image must always be less than the corresponding point in the "first-echo" image, except under certain circumstances to be enumerated below. This fact is not obvious while viewing the images on the display consoles or photographic film, since the system "normalizes" the intensity of each image to improve viewabili- 


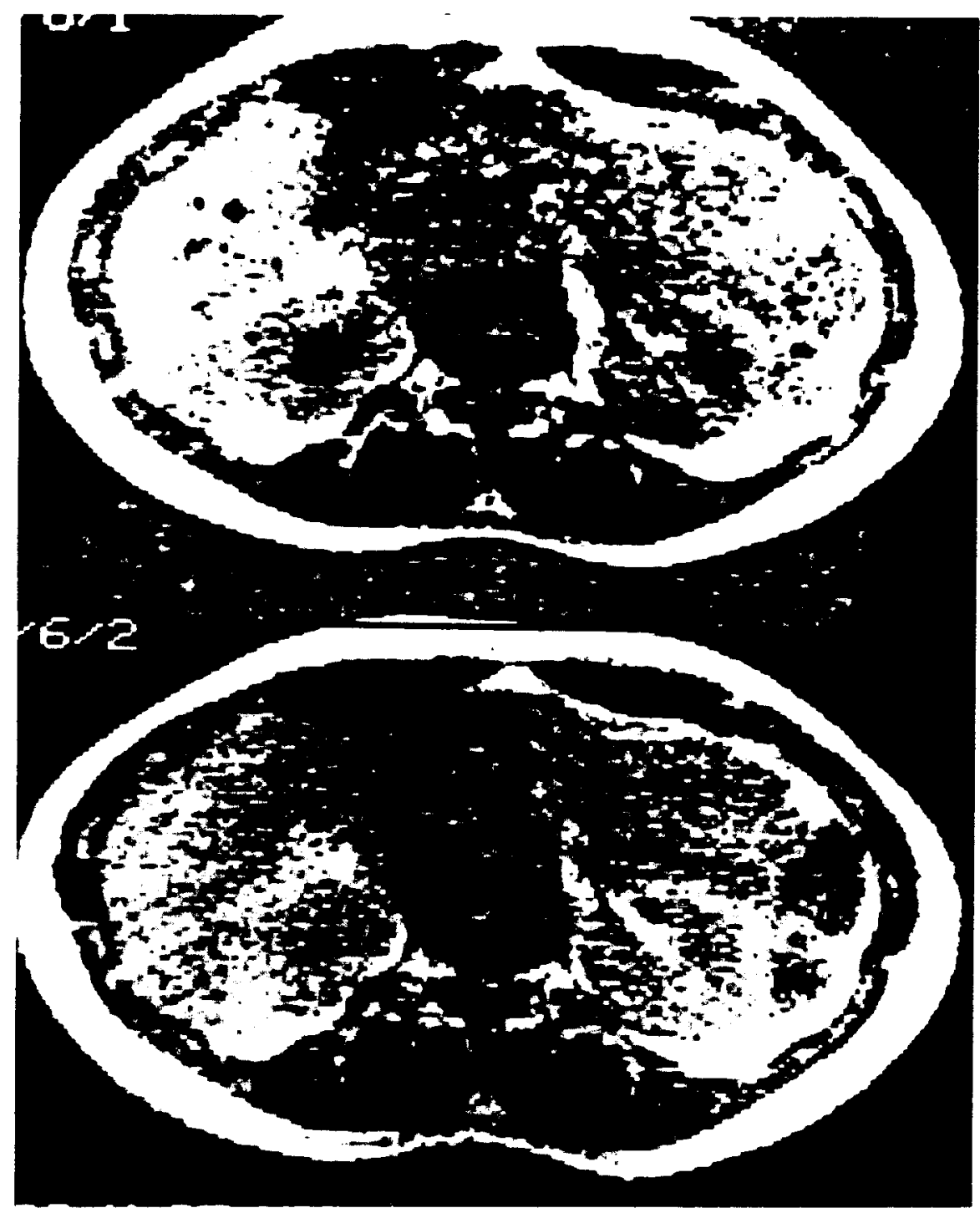

Fig. 2. First echo (top) and second echo (bottom) images of patient with retroperitoneal fibrosis, with intensity normalization suppressed. $T R=0.5 \mathrm{sec}$., and $T E=28$ and $56 \mathrm{msec}$, respectively. Both the kidneys and the paraspinal muscles in the first echo image are of diminished relative intensity.

ty; thus the displayed intensity of the second-echo images are increased relative to the first. Measurements of renal relaxation times were made using regions of interest which included both renal medulla and renal cortex; the hilar structures were excluded. In reviewing these calculations we noted several with "negative" $T 2$ values.

To determine approximately how often such anomalous data will occur in routine clinical scanning, we reviewed images for 101 patients ( 54 were selected randomly; the remainder were available from an unrelated study). In 55 patients, both kidneys werc examined, and in the majority of patients data were available from two imaging sequences with different $T R$ intervals. Thus, intensities were measured and $T 2$ values calculated for a total of 220 kidney images.

To further investigate such phenomena, 5 normal volunteers underwent upper abdominal MRI before and immediately after 5 to 10 minutes of vigorous exercise, employed to increase both blood flow and respiratory motion. Transverse images were obtained at a TR of $0.5 \mathrm{sec}$.

\section{RESULTS}

The results on the patients in whom the reversal of second echo intensity was noted are summarized in Table 1. The first patient had marked renal disease 


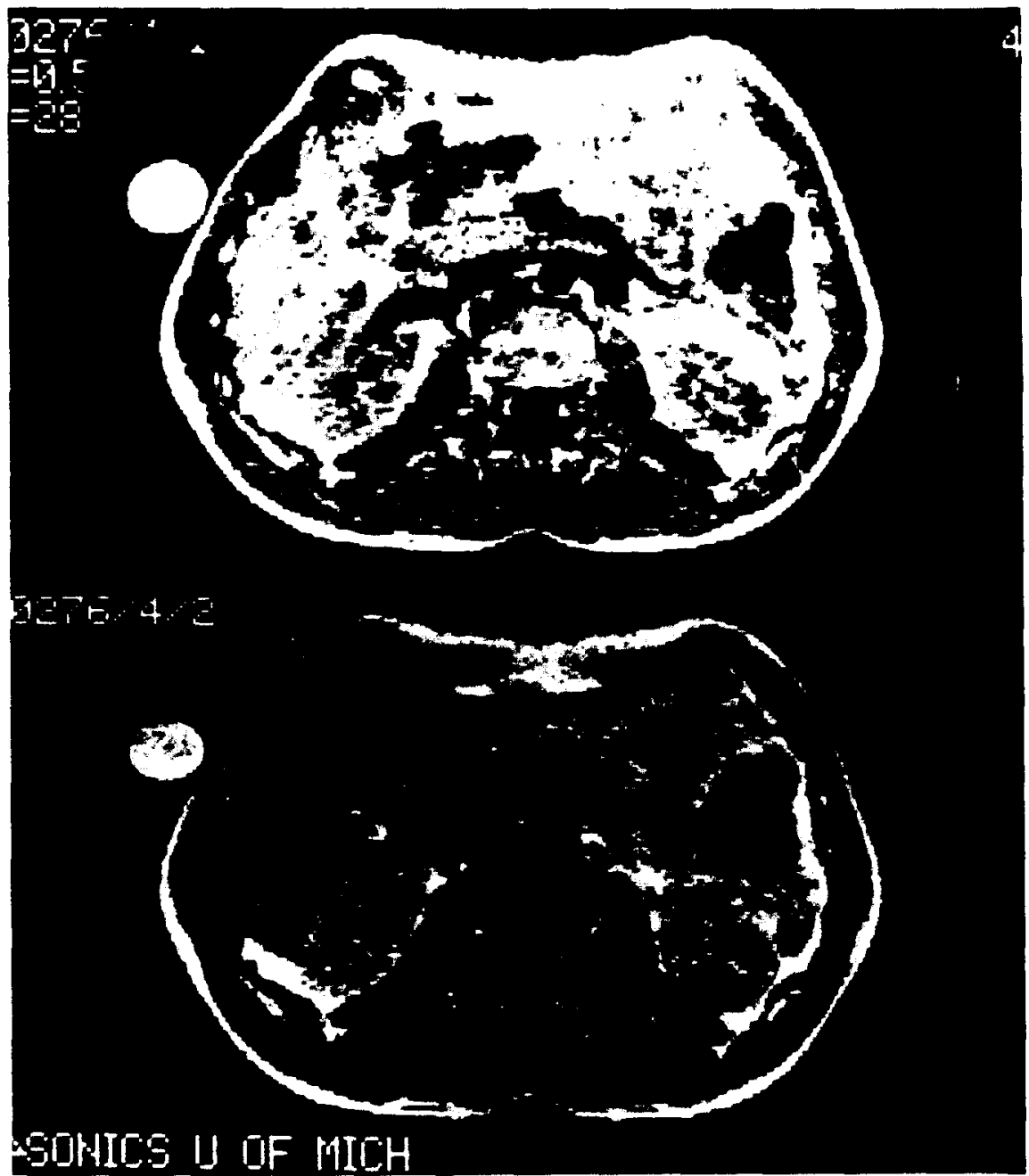

Fig. 3. First echo (top) and second echo (bottom) images in a normal volunteer prior to exercise, with intensity normalization suppressed. $T R=0.5 \mathrm{sec}, T E=28$ and 56 msec, respectively. The renal parenchymal signal is greater on the first echo image than on the second.

secondary to systemic lupus erythematosus; the second had retroperitoneal fibrosis without evidence for renal disease; and the third had a hepatic hemangioma.

In the patient with SLE, the anomalous intensity values were limited to the kidneys. The patient was felt to have a hyperdynamic circulation by the clinical service, and the possibility that either a motion effect or possibly a flow phenomenon might be present was considered. The scan did not show obvious motion artifact, but respiratory movement of the kidneys, especially in the cranial-caudad direction perpendicular to the image plane, may certainly have been present. Figure 1 demonstrates the first and second echo images, showing inversion of the expected MR intensities for both kidneys; no such effect was observed in the other tissues, which served as controls.
In the second patient, intensity inversion was limited to the left kidney (Table 1) and the images did show artifacts caused by respiratory motion (Fig. 2). In the third patient, the inversion was noted in the normal hepatic parenchyma of the dome of the liver.

Inverted intensities and negative calculated $\mathrm{T} 2$ values did not occur in any of the 101 patient sample. For these patients, the mean calculated $T 2$ value of the kidneys was 69 , with a standard deviation of 62 $(n=220)$.

All five volunteers had unremarkable pre-exercise images without intensity inversion. On post-exercise images, however, two volunteers demonstrated an inversion in the relative intensities of the renal parenchyma on the first and second echo images; whereas the paraspinal muscles and liver did not show the 


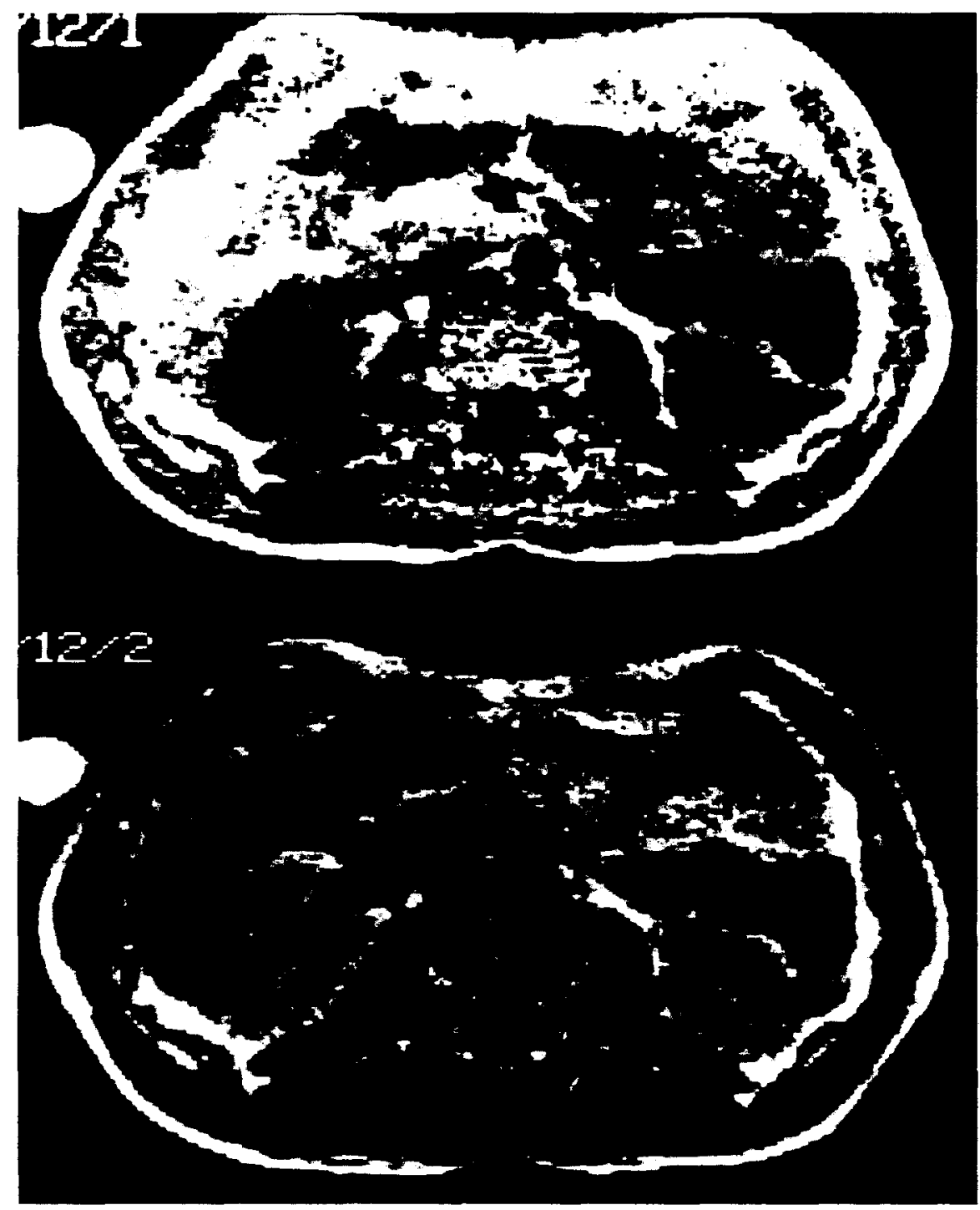

Fig. 4. First echo (top) and second echo (bottom) images in the same normal volunteer after exercise, again with intensity normalization suppressed. $T R=0.5 \mathrm{sec}$, $T E=28$ and $56 \mathrm{msec}$, respectively. The renal parenchymal signal in the first echo image is diminished compared to the surrounding structures, and compared to the second echo image.

effect. The anomalous data appear to result from a peculiar lowering of the renal intensity on the first echo image. Figure 3 shows the first and second echo images of volunteer 1 before exercise; figure 4 is a postexercise image. Intensity calculations from the TR = $0.5 \mathrm{sec}$ data show very significant quantitative differences between the kidneys (Table 2). Table 2 also reports data for volunteer 2 whose kidneys also exhibited intensity reversal.

\section{DISCUSSION}

Our data clearly shows anomalous intensity reversal (higher intensity on second echo images) within organ parenchyma using spin-echo MR imaging; calculated T2 values from such anomalous intensity images would give spurious, negative values. The signal intensity on second echo images can be greater than on the first echo in the following circumstances:

1. when flow effects are present

2. when there is patient motion

3. due to random, statistical variation in the intensities; this is most apparent when the intensities are measured using small regions of interest.

4. when the imager is mistuned, malfunctioning (e.g., abnormally low intensity first echo images), or when artifacts are present. 
Table 2. Magnetic resonance intensities pre- and post-exercise

\begin{tabular}{|c|c|c|c|c|c|c|c|c|}
\hline \multirow[b]{2}{*}{ Organ } & \multicolumn{4}{|c|}{ Pre-exercise } & \multicolumn{4}{|c|}{ Post-exercise } \\
\hline & $I_{1}(\mathrm{TE}-28 \mathrm{msec})$ & $\begin{array}{l}\text { Intensities } \\
I_{2}(\mathrm{TE}-56 \mathrm{msec})\end{array}$ & $I_{1} / I_{2}$ & $T_{2}$ & $I_{1}(\mathrm{TE}=28 \mathrm{msec})$ & $\begin{array}{c}\text { Intensities } \\
I_{2}(\mathrm{TE}-56 \mathrm{msec})\end{array}$ & $I_{1} / I_{2}$ & $T_{2}$ \\
\hline \multicolumn{9}{|l|}{ Volunteer 1} \\
\hline left kidney & 1974 & 1435 & 1.38 & 88 & 1121 & 1169 & 0.96 & -668 \\
\hline left paraspinal muscle & 1483 & 625 & 2.37 & 32 & 1668 & 602 & 2.77 & 28 \\
\hline right kidney & 1858 & 1474 & 1.26 & 121 & 592 & 1151 & 0.51 & -42 \\
\hline right paraspinal muscle & 1437 & 607 & 2.37 & 32 & 1133 & 380 & 2.98 & 26 \\
\hline liver & 2247 & 1091 & 2.05 & 39 & 2089 & 1121 & 1.86 & 50 \\
\hline \multicolumn{9}{|l|}{ Volunteer 2} \\
\hline left kidney & 1689 & 1153 & 1.47 & 73 & 959 & 981 & 0.98 & -1234 \\
\hline left paraspinal muscle & 1062 & 433 & 2.45 & 31 & 1298 & 512 & 2.53 & 30 \\
\hline right kidney & 1872 & 1170 & 1.60 & 60 & 530 & 821 & 0.64 & -64 \\
\hline right paraspinal muscle & 1181 & 426 & 2.77 & 28 & 1009 & 408 & 2.47 & 31 \\
\hline liver & 1659 & 972 & 1.71 & 52 & 1217 & 706 & 1.72 & 51 \\
\hline \multicolumn{9}{|c|}{ Volunteer 2, level several centimeters cranial to above slice } \\
\hline left kidney & 1747 & 993 & 1.76 & 50 & 699 & 718 & 0.97 & -1044 \\
\hline left paraspinal muscle & 849 & 304 & 2.79 & 27 & 1267 & 474 & 2.67 & 29 \\
\hline right kidney & 1425 & 809 & 1.76 & 50 & 410 & 967 & 0.42 & -32 \\
\hline right paraspinal muscle & 1125 & 452 & 2.49 & 31 & 1103 & 438 & 2.51 & 30 \\
\hline anterior liver & 1790 & 978 & 1.83 & 46 & 953 & 679 & 1.40 & 83 \\
\hline posterior liver & 1696 & 898 & 1.89 & 44 & 556 & 592 & 0.94 & -446 \\
\hline
\end{tabular}

Note: Pre- and post-exercise data are taken from approximately the same anatomic level; slice numbers in the multi-slice imaging sequence differed due to slight discrepancies in repositioning the subjects on the scanner table. TR $=0.5 \mathrm{sec}$.

Molecular diffusion effects may also affect T2 measurements, though they are likely to produce falsely low values."

Statistical fluctuations can be excluded since the observed effects involved large areas, and imager malfunction was also discounted by examination of phantom and patient head and low pelvis images. In review- ing all of the available data, respiratory motion, particularly in the cranial-caudad direction, seems the most likely explanation for the intensity reversal we report. The asymmetry of the intensity inversion would be explained by greater respiratory motion of the left kidney than the right. Blood flow effects ${ }^{5-7}$ cannot be excluded as an explanation of the anomalous intensi-

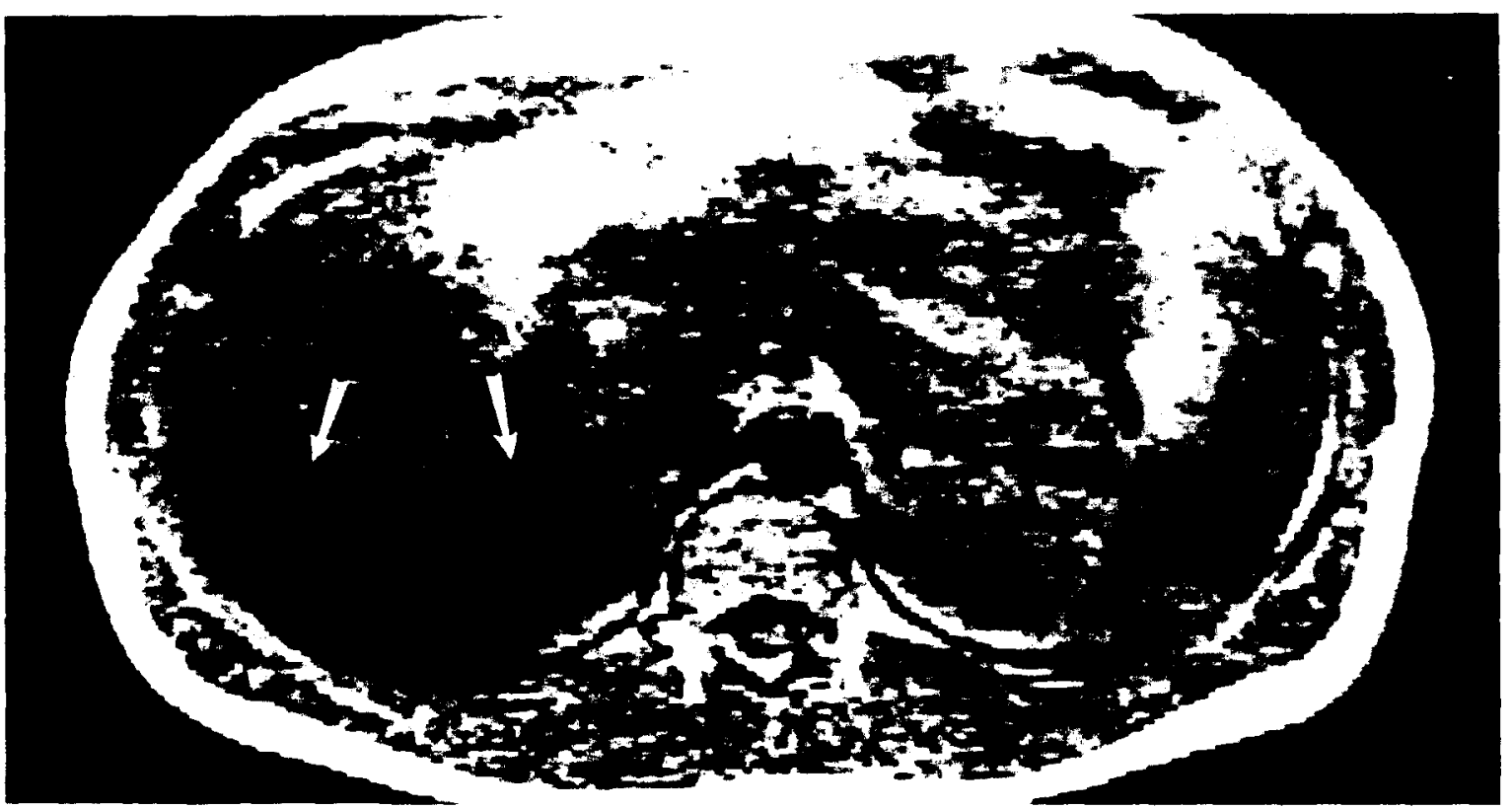

Fig. 5. First echo image of the second volunteer, post-exercise. $T R=0.5 \mathrm{sec}, T E=28 \mathrm{msec}$. Note the wedge shaped area of diminished intensity in the posterior portion of the liver (arrows), as well as the diminished intensity of both kidneys. 
ties, though they scem unlikely to be an adequate explanation given their asymmetric distribution.

Whatever its origin, the intensity reversal phenomenon carries important implications for MR imaging, as it introduces inaccuracies in the quantitation of the magnetic parameters. Such measurements have been suggested to be diagnostically useful. ${ }^{89}$ Even in those patients without actual intensity reversals, the reliability of in vivo relaxation time determinations from ungated, dual spin-echo images is likely to be limited, judging by the wide variation we encountered in our 101 patient sample. Routine abdominal MRI scans are performed with patients as relaxed as possible; nonetheless we have noted obvious areas of anomalous intensity in a small percentage of the studies. Generally, these are crescent shaped regions of the liver or involve the kidneys in a manner similar to the cases described here. Presumably, respiration in these individuals is greater than with most patients. It seems likely that cranial-caudad respiratory motion of the kidneys, which may well not be obvious on the images, can be a source of considerable error. It should, however, be possible to correct for such effects, through the use of respiratory gating, different pulse sequences, or improved algorithms for image quantitation. More work is clearly required, if optimal use is to be made of measured relaxation times in the upper abdominal viscera.

\section{REFERENCES}

1. Wood, M. and Henkelman, R.M. NMR image artifacts from periodic motion. Magn. Reson. Med. 1:275, 1984.

2. Schultz, C.L.; Alfidi, R.J.; Nelson, A.D.; Kopiwoda, S.Y.; Clampitt, M.E. The effects of motion on twodimensional fourier transformation magnetic resonance images. Radiology 151:117-122; 1984.

3. Crooks, L.; Arakawa, M.; Hoenninger, J. et al. Nuclear magnetic resonance whole-body imager operating at 3.5 kGauss. Radiology 143:169-174; 1982.

4. Wesby, G.; Moon, K.; Crooks, L.; Arakawa, M.; Brasch, $R$. Proton T2 reduction due to spin-diffusion through pulsed-gradient in spin-echo NMR imaging. Imaging implications and applications. Magn. Reson. Med. 1:273 $274 ; 1984$.

5. Mills, C.M.; Brant-Zawadzki, M.; Crooks, L.E.; Kauf- man, L.; Seldon, P.; Norman, D.; Bank, W.; and Newton, T.H. Nuclear magnetic resonance: principles of blood flow imaging. AJNR 4:1161-1166; 1983.

6. Walluch, V. and Bradley, W.G. Even-echo rephasing in slow laminar flow. J. Comput. Assist. Tomogr. (in press).

7. Axel, L. Blood flow effects in magnetic resonance imaging. AJR 143:1157-1166; 1984.

8. Smith F.W.; Reid, A.; Mallard, J.R.; Hutchison, J.M.S.; Power, D.A.; Catto, G.R.D. Nuclear magnetic resonance imaging in renal disease. Diagnostic Imaging 51:209 $213 ; 1982$.

9. Hricak, H.; Crooks, L.; Sheldon, P.; and Kaufman, L. Nuclear magnetic resonance imaging of the kidney. Radiology 146:425-432; 1983. 